\title{
Technologie de production simultanée de purée et du jus de tomate
}

\author{
Paul Ayihadji Ferdinand HOUSSOU ${ }^{1 *}$, Valère DANSOU ${ }^{1}$, Lucie AYI-FANOU $^{2}$, \\ Abdelrahim Djimet ABDELKERIM ${ }^{3}$ et Guy Apollinaire MENSAH ${ }^{1}$ \\ ${ }^{1}$ Programme Technologies Agricole Alimentaire de l'Institut National des Recherches Agricoles du Bénin 01 \\ BP 128 Porto-Novo; République du Bénin. \\ ${ }^{2}$ Laboratoire de Biochimie et de Biologie Moléculaire, Faculté des Sciences et Techniques, \\ Université d'Abomey-Calavi, 04 BP 0320 Cotonou, République du Benin. \\ ${ }^{3}$ Université Africaine de Technologie et de Management UATM/GASA Formation 04 BP 1361 Cotonou; \\ République du Bénin. \\ *Auteur correspondant ; E-mail : houssou02@yahoo.fr; BP : 128 Porto-Novo, Tél : (+229) 97886951.
}

\section{RESUME}

La tomate est un produit maraîcher très important dans l'alimentation humaine en Afrique et dans le monde. L'objectif de l'étude est d'évaluer une nouvelle méthode de transformation simultanée de la tomate en purée et en jus comestible. La durée de la production, la quantité d'énergie et les rendements de la purée et du jus ont été évalués. L'ancienne méthode permet d'avoir uniquement la purée par évaporation et concentration. Avec la nouvelle méthode, la purée, le jus: salé à $1 \%$ et non salé ont été produits puis caractérisés sur le plan organoleptique et nutritionnel surtout pour le jus le plus apprécié par les participants. Les résultats obtenus montrent que pour transformer $15 \mathrm{~kg}$ de tomate, la durée de cuisson et la consommation en énergie pour la méthode 1 et 2 sont respectivement de 1 heure et 4 heures de $3 \mathrm{~kg}$ et 8,5 $\mathrm{kg}$ de charbon. La nouvelle méthode a donné un rendement en purée de $22,66 \%$ contre $24,33 \%$ pour l'ancienne méthode. Le test sensoriel a révélé que $70 \%$ des participants ont apprécié le jus salé et $30 \%$ pour le jus non salé. Les teneurs en vitamines $\mathrm{C}$ et $\beta$ carotènes sont presque les mêmes pour les deux jus.

(C) 2015 International Formulae Group. All rights reserved.

Mots clés : Tomate, transformation, purée, jus.

\section{Technology of simultaneous production of tomato purée and juice}

\begin{abstract}
Tomato is a very important gardening product in the human food in Africa and in the world. The aim of the study is to evaluate a new method of tomato process simultaneously tomato into purée and edible juice. The parameters evaluated were: production time, energy used and yields of purée and juice obtained. The former method allows only for the puree production through evaporation and concentration. With the new method, in addition to purée of tomato, two types of juice: $1 \%$ salted and unsalted were produced and characterized for sensory aspects and nutritional only for the juice mostly appreciated by panelists. The results indicated that to process $15 \mathrm{~kg}$ of tomatoes, the cooking time is one hour with low power consumption $3 \mathrm{~kg}$ of charcoal for the new method while for the former method, the duration is 4 hours and $8.5 \mathrm{~kg}$ of charcoal used. The new method
\end{abstract}


gave $22.66 \%$ of purée yield against $24.33 \%$ for the old method. The sensory test revealed that $70 \%$ of participants liked the salted juice and $30 \%$ unsalted juice. The contents in vitamin $\mathrm{C}$ and $\beta$-carotenes are nearly the same for the two juices.

(C) 2015 International Formulae Group. All rights reserved.

Keywords: Tomato, processing, purée, juice.

\section{INTRODUCTION}

La tomate est l'un des fruit-légumes les plus consommés et très importante dans l'alimentation dans le monde entier (Boumendjel et al., 2012). Au plan nutritionnel, c'est une source de sels minéraux tels $\mathrm{Ca}, \mathrm{K}, \mathrm{Mg}, \mathrm{Na}, \mathrm{Fe}$ et de vitamines comme A, B6, C, E (Boumendjel et Boutebba, 2003 ; Glouchkoff, 2010 ; Sawadogo et al., 2015). Ce fruit est très fragile et périssable à cause de sa teneur en eau qui est supérieure à $85 \%$, ce qui est souvent à l'origine des pertes post-récolte. Ces pertes peuvent atteindre plus de $40 \%$ dans beaucoup de pays Ouest africains où les industries modernes de production de concentré de tomates sont presque inexistantes (Dossou et al., 2007). Au Benin comme dans de nombreux pays de l'Afrique de l'Ouest, la transformation artisanale de la tomate en purée est la forme de transformation /conservation la plus répandue pour réduire ces pertes post-récolte (Montcho, 2002). Mais cette transformation nécessite l'utilisation d'énorme quantité d'énergie (charbon de bois ou de gaz combustible) pour faire évaporer suffisamment l'eau contenue dans la tomate (Abdelkerim, 2014). Par ailleurs, la production du jus de tomate est inexistante ou très peu connue dans nos pays Ouest africains; en particulier, le Bénin. Dans les pays développés, ce jus de tomate est utilisé à la fois comme boisson ou comme condiment dans les restaurants (Abdelkerim, 2014). L'objectif de cette étude est de développer une nouvelle méthode de transformation de la tomate en purée et qui permet en même temps de récupérer son eau par pressage pour en faire le jus. La mise au point de cette technologie permettra aux femmes transformatrices et aux petites unités de production de purée, de mieux valoriser la transformation de la tomate et de réduire de façon significative la quantité d'énergie utilisée pour évaporer l'eau lors de la cuisson de la purée.

\section{MATERIEL ET METHODES Matériel végétal}

Le cultivar de tomate fruit (Lycopersicon esculentum) dénommé "Akikonkoui" (Figure 1) en langue locale fongbé au Bénin a été utilisée pour la production du jus et de purée de tomate. Cette variété est l'une des variétés locales les plus cultivées et consommée par les populations au sud du Benin (Kpagbin, 2011).

\section{Equipement utilisé}

Le principal équipement qui a été utilisé pour la transformation de la tomate en purée et en jus a été l'épépineuse motorisée "complexe tomate plus" (Figure 2). Cet équipement a été mis au point au niveau du Programme Technologies Agricole et Alimentaire (PTAA) de l'Institut National des Recherches Agricoles du Bénin (INRAB). Il permet la mouture de la tomate en y enlevant la peau et les pépins. La peau et les pépins constituent les sous-produits; seul le moût est utilisé dans la chaîne de production de la purée et du jus.

\section{Production du jus et de la purée de tomate}

La nouvelle méthode de production simultanée de purée et du jus de tomate (méthode1) a été réalisée en suivant le diagramme technologique de la Figure 3. L'ancienne pratique de production unique de purée de tomate (méthode 2) décrite par Kiki et Fagbohoun (1999) a été utilisée comme 
témoin. Pour la méthode1, le jus de tomate a été extrait par tamisage et pressage (essorage) du moût chauffé pendant $10 \mathrm{~min}$. Le moût, après extraction du jus, a été cuit pour obtenir la purée. Le jus récupéré par pressage a été ensuite pasteurisé. Après pasteurisation, le jus a été divisé en deux, une moitié a été salée à $1 \%$ en ajoutant du sel de cuisine. Chaque type de jus a été conditionné dans des bouteilles stérilisées de $30 \mathrm{cl}$. Quant à la deuxième (méthode 2), le moût obtenu après la mouture de la tomate a été directement cuit pour l'obtention de la purée de tomate. Chaque production a été répétée trois fois.

\section{Détermination des paramètres technologiques}

Durée des opérations :

Lors du processus de transformation de la tomate en purée et en jus, la durée des opérations a été déterminée en considérant le temps du début et de la fin de chaque opération unitaire.

Rendement de production :

Le rendement de production a été déterminé par le rapport entre la quantité du jus ou de la purée de tomate obtenue sur la quantité de tomates transformée suivant la formule suivante :

Pour la purée

$$
R p=\frac{\text { poids de la purée }}{\text { poids de tomate utilisée }} \times 100
$$

$R p$ : Rendement de la production de purée. Pour le jus :

$$
R j=\frac{\text { poids du jus obtenu }}{\text { poids de tomate utilisée }} \times 100
$$

$R j$ : Rendement de production de jus.

Consommation d'énergie pour production :

Le charbon de bois a été utilisé comme source d'énergie pour la production de purée et du jus de tomate au cours des opérations de préchauffage, de cuisson et de la pasteurisation. La consommation en charbon de bois a été évaluée en pesant le poids du charbon avant et après chacune de ces opérations.

\section{Evaluation de la qualité organoleptique des jus de tomate}

Six litres de jus de tomate issus de la nouvelle technologie (méthode 1) de production simultanée de jus et de la purée de tomate ont été divisés en deux lots de 3 litres. Le premier lot de trois litres a été salé à $1 \%$ de sel cuisine (jus A) et le second lot non salé (jus B). Des échantillons de $100 \mathrm{ml}$ contenus dans des verres en plastique jetables ont été soumis à l'appréciation de 30 dégustateurs bien entrânés. Ceux-ci étaient chargés d'apprécier la qualité des jus par rapport à la couleur, l'odeur et le goût en utilisant une échelle d'appréciation de : 1=Aime beaucoup ; $2=$ Aime un peu et $3=$ n'aime pas du tout.

\section{Evaluation de la qualité nutritionnelle du jus de tomate}

Dans cette étude, seul le jus a été analysé car il constitue le nouveau produit. Les paramètres évalués ont été : la matière sèche soluble, la teneur en vitamines $C$ et $\beta$ carotène ainsi que la teneur en potassium.

Matière sèche soluble: La matière sèche soluble de la purée a été déterminée à l'aide d'un réfractomètre portatif (PARALUX, France) gradué de 0 à $50^{\circ}$ Brix. Une goutte d'échantillon humide a été déposée sur la lentille du réfractomètre et la lecture a été faite directement après exposition à la lumière.

Dosage de la vitamine $C$ et de la $\beta$ carotène: La teneur en vitamine $\mathrm{C}$ a été déterminée par titration avec une solution de 2,6-dichlorophénol-indophénol selon la méthode AOAC (2012). Les résultats ont été exprimés en $\mathrm{mg} / 100 \mathrm{~g}$ de jus. La $\beta$-carotène a été dosée par la méthode décrite par Biehler et al. (2009).

Dosage de sodium et de potassium : Le potassium a été déterminé par photométrie de flamme d'après la méthode décrite par Gueguen et Rombauts (1961). 


\section{Analyse statistique des données}

Les résultats d'analyse physicochimique, sensorielle et nutritionnelle ont été traités avec le logiciel Microsoft Excel. L'analyse de variance à un critère (ANOVA One-way), à l'aide du logiciel SPSS v16.0 a été réalisée pour tester la significativité de la différence entre les moyennes à un seuil de $5 \%$ pour chaque paramètre.

\section{RESULTATS}

Production simultanée du jus et de la purée de tomate

$\begin{array}{cccc}\begin{array}{c}\text { Les Figures } \\ \text { respectivement }\end{array} & 3 \text { et } 4 & \text { représentent } \\ \text { les } & & \text { diagrammes }\end{array}$ technologiques de production de la purée de tomate suivant l'ancienne méthode (méthode 1) et celle de la nouvelle méthode (méthode 2). Les résultats obtenus montrent que la durée de cuisson (4 heures) et la quantité de charbon consommée $(8,5 \mathrm{~kg})$ pour la méthode 1 sont significativement supérieures $(\mathrm{p}<0,05)$ respectivement à la durée de cuisson (1 heure) et à la quantité de charbon consommée $(3 \mathrm{~kg})$ utilisées par la méthode 2 (Tableau 1). Par contre, le rendement en purée $(24,33 \%)$ et en matière sèche soluble $(19,1 \%)$ de la purée issue de la méthode 1 (Tableau 1) sont significativement supérieurs $(\mathrm{p}<0,05)$ à ceux de la nouvelle méthode $2(22,66 \%$ et $15,8 \%)$. Mais avec cette nouvelle méthode, en plus de la purée, un rendement de $42,07 \%$ de jus de tomate a été obtenu.

\section{Qualité organoleptique du jus de tomate}

A l'issue des tests de dégustation, les résultats obtenus montrent que parmi les deux types de jus (Jus A et jus B) soumis à l'appréciation des dégustateurs, $75 \%$ des participants ont choisi les deux types de jus en considérant principalement la couleur et l'odeur agréables de chacun des deux échantillons (Figure 5). Par contre, le goût a été le facteur de différenciation des deux jus. Soixante-dix pourcent (70\%) des dégustateurs n'ont pas aimé le jus non salé (jus B) (Figure 5) et l'ont rejeté au profit du jus salé.

\section{Qualité nutritionnelle des deux types de jus de tomate}

Les résultats de l'analyse nutritionnelle de chacun des deux types de jus de tomate montrent que la teneur en $\beta$-carotènes du jus salé $(478 \mu \mathrm{g} / 100 \mathrm{ml})$ semble être relativement plus élevée que celle du jus non salé (368 $\mu \mathrm{g} / 100 \mathrm{ml})$. En ce qui concerne la concentration en potassium et en vitamine $C$, aucune différence significative $(p>0,05)$ n'a été observée pour le jus salé (A) et le jus non salé B (Tableau 2).

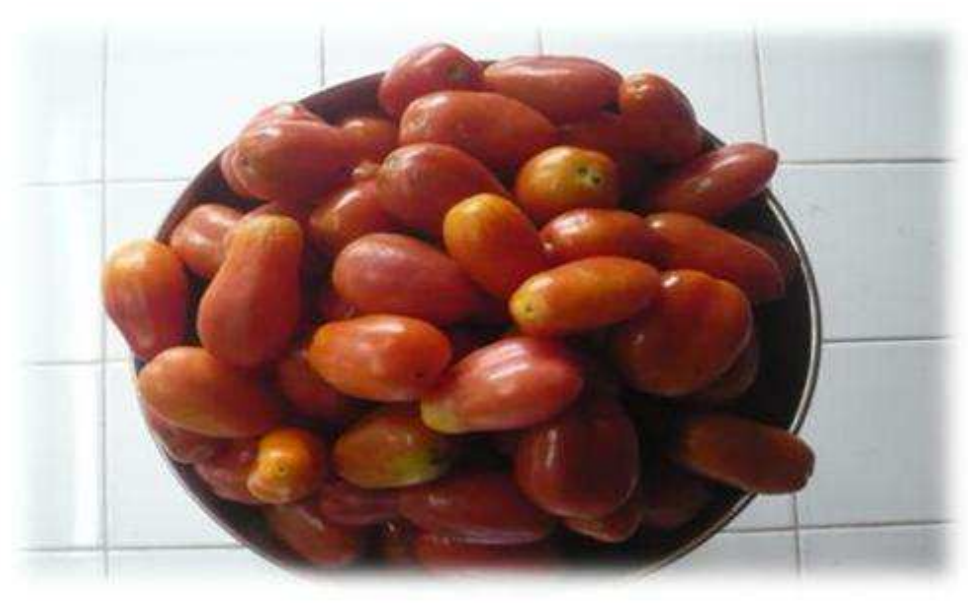

Figure 1 : Fruit de tomate (Lycopersicon esculentum) dénommé Akikonkoui en langue locale au Bénin. 


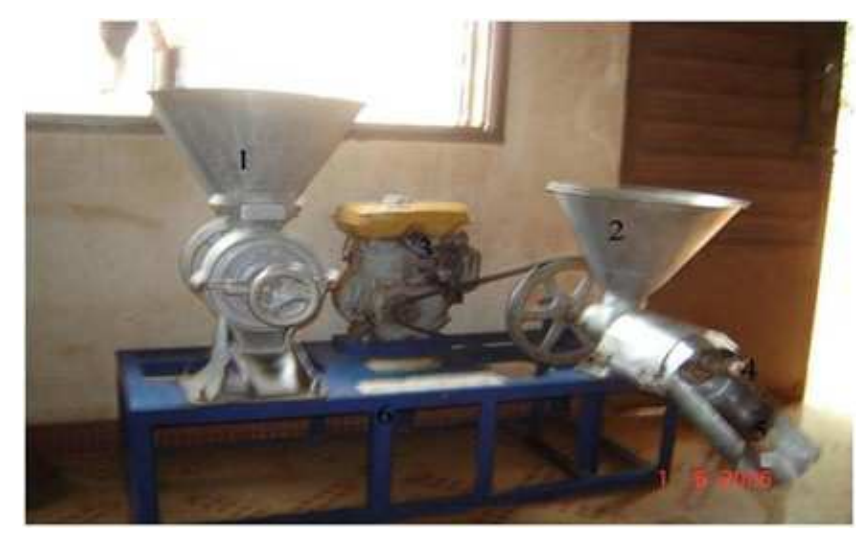

Figure 2 : Epépineuse motorisée "'Tomate plus".

Trémie (01) ; Trémie (02); Moteur robin(3); Vise sans fin(4) ; Cône d'évacuation(5); Chassis de l'épépineuse(6)

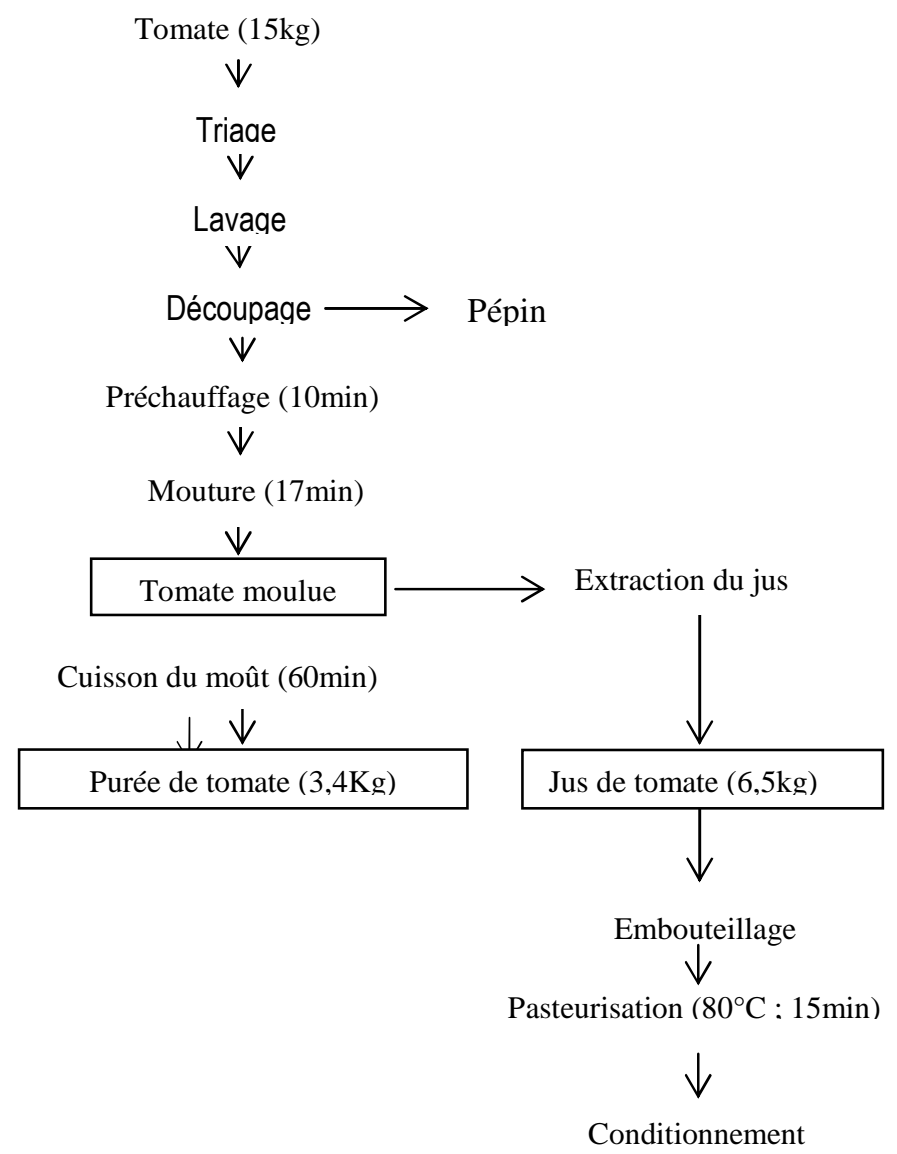

Figure 3 : Production simultanée de la purée et de jus de tomate. 


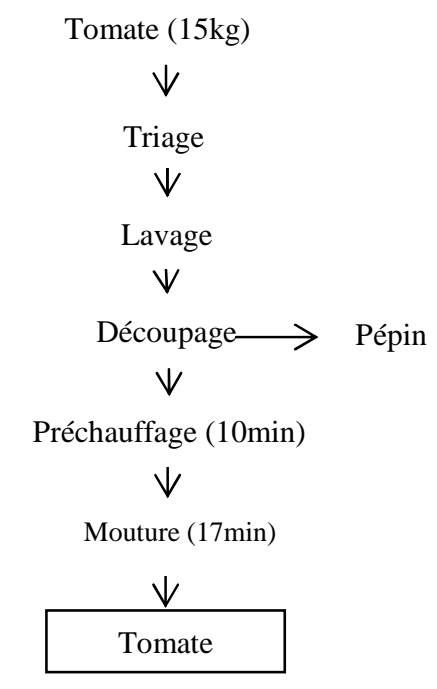

Cuisson du moût (4h)

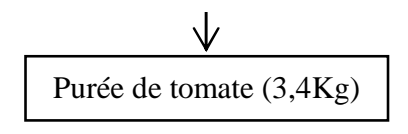

Figure 4: Production de la purée de tomate.

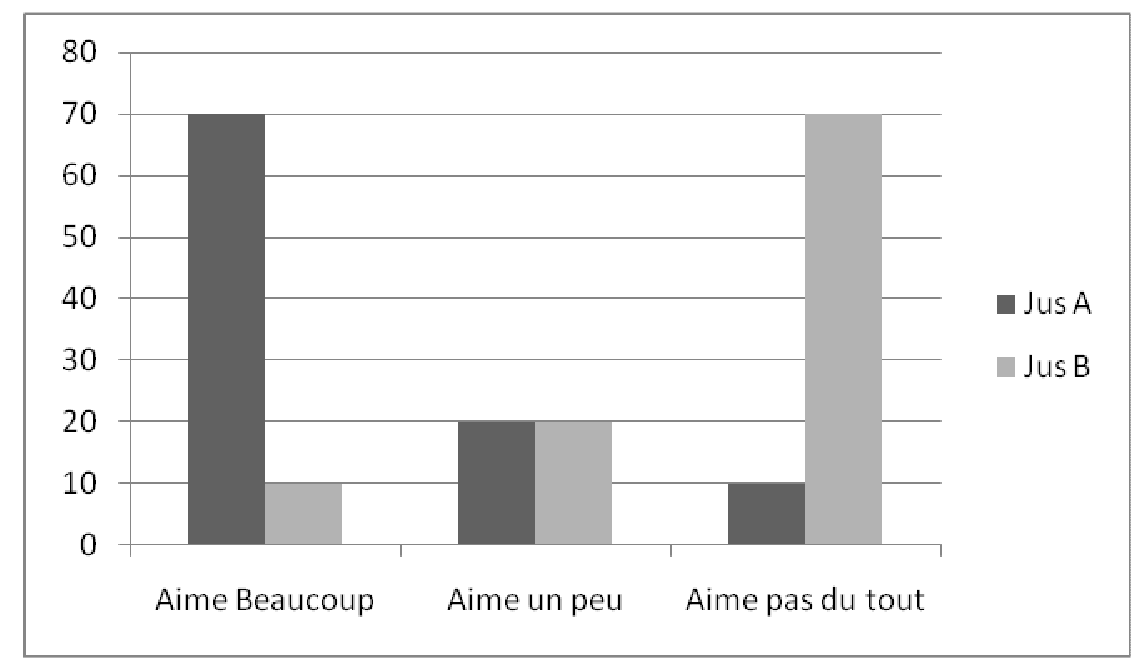

Figure 5 : Préférence des dégustateurs des jus de tomates. Jus A : Salé ; Jus B non salé. 
Tableau 1 : Paramètre technologique de production de purée et de jus de tomate.

\begin{tabular}{lccccc}
\hline & $\begin{array}{c}\text { Rendement } \\
\text { en jus }(\boldsymbol{\%})\end{array}$ & $\begin{array}{c}\text { Rendement } \\
\text { en purée } \\
\mathbf{( \% )}\end{array}$ & $\begin{array}{c}\text { Matière sèche } \\
\text { soluble purée } \\
(\boldsymbol{\%})\end{array}$ & $\begin{array}{c}\text { Durée de } \\
\text { cuisson purée } \\
(\text { heure })\end{array}$ & $\begin{array}{c}\text { Consommation } \\
\text { en énergie purée } \\
(\mathbf{k g})\end{array}$ \\
\hline Méthode 2 & 42,07 & $22,66 \mathrm{a}$ & $15,8 \mathrm{a}$ & $1 \mathrm{a}$ & $3 \mathrm{a}$ \\
Méthode 1 & --- & $24,33 \mathrm{~b}$ & $19,1 \mathrm{~b}$ & $4 \mathrm{~b}$ & $8,5 \mathrm{~b}$ \\
\hline
\end{tabular}

Les moyennes portant les lettres différentes dans la même colonne sont significativement différentes au seuil de $5 \%$.

Méthode 1 : méthode traditionnelle de préparation de purée de tomate par évaporation d'eau.

Méthode 2 : nouvelle méthode de préparation simultanée de purée et de jus de tomate.

Tableau 2 : Composition nutritionnelle des deux type de jus A et B.

\begin{tabular}{lccc}
\hline Paramètres & $\begin{array}{c}\text { Concentration en } \\
\text { potassium }(\mathbf{m g})\end{array}$ & $\begin{array}{c}\text { Taux de la } \\
\text { vitamine } \mathbf{C}(\mathbf{m g})\end{array}$ & $\begin{array}{c}\text { Taux de } \boldsymbol{\beta} \\
\text { carotènes }(\boldsymbol{\mu g})\end{array}$ \\
\hline Jus de tomate A & $232 \mathrm{a}$ & $9,41 \mathrm{a}$ & $478 \mathrm{~b}$ \\
Jus de tomate B & 231a & $10,46 \mathrm{a}$ & $368 \mathrm{a}$ \\
\hline \multicolumn{2}{c}{ Les moyennes portant les lettres différentes dans la même colonne sont significativement différentes $(\mathrm{p}<0,05)$. Jus } \\
A : salé ; jus B : non salé.
\end{tabular}

\section{DISCUSSION}

La transformation de la tomate en purée est une solution alternative dans la réduction des pertes post-récolte (Montcho et Ahouansou, 2004 ; Dossou et al., 2006). La comparaison de la méthode (méthode 2) de production simultanée de purée et de jus de tomate à celle habituellement utilisée qui consiste à la transformation de la tomate en purée uniquement (méthode 1) indique que cette dernière est moins performante en considérant la durée de cuisson et la consommation en énergie utilisées pour la préparation de la purée (Tableau 1). En effet pour la méthode 2 , la purée a été pressée par essorage et filtrage pour récupérer le jus. Ce jus obtenu n'étant pas limpide, contient encore quelques résidus de purée, ce qui explique le rendement et la matière sèche relativement faibles obtenus pour cette méthode. D'après Montcho (2002), le rendement en purée de tomate est lié d'une part à la variété de tomate mais aussi à la technologie utilisée. Par ailleurs, les rendements de production en purée de la méthode $2(22,66 \%)$ et méthode 1 $(24,33 \%)$ sont supérieurs à celui obtenu
(20,69\%) par Montcho (2002) qui a utilisé une autre variété de tomate (tounvi). Dans le cadre de cette étude, une corrélation positive est observée entre la durée de cuisson de la purée de tomate des méthodes 1 et 2 par rapport à la consommation en énergie. Une différence significative est notée entre les deux méthodes par rapport à la durée de cuisson de la purée ainsi que la consommation en énergie des méthodes 1 et 2 (4 heures contre 1 heure et $8,5 \mathrm{~kg}$ de charbon contre 3 $\mathrm{kg}$ ). Cette différence est due au fait que le moût obtenu a été essoré pour extraire le jus, ceci après quelques minutes de cuisson. Cette réduction de jus par essorage a contribué à diminuer le temps de cuisson de la purée (méthode 2). Par conséquent, avec la méthode 2, un gain substantiel de temps dans la production de la purée est réalisé avec une faible consommation d'énergie par rapport à l'ancienne méthode 1. De plus, la nouvelle méthode présente l'avantage, d'avoir en plus de la purée, de jus de tomate avec un rendement de $42,07 \%$ par rapport à la méthode 1 . 
Sur le plan d'analyse sensorielle, la qualité organoleptique du jus A qui est très appréciée des dégustateurs grâce à de son goût légèrement salé par rapport au jus B non salé qui a un goût fade selon les dégustateurs. Par ailleurs, la couleur de ces deux jus est un peu claire, caractéristique des jus de fruits connus par les consommateurs comme les jus d'ananas, de mangue et de goyave. De même, la préférence des dégustateurs est aussi motivée par l'odeur attirante et moins prononcée de ces jus de tomate. En dehors de cette motivation, plusieurs avantages du point de vue nutritif s'offrent aux consommateurs du jus de tomate salé en l'occurrence. Du point de vue de la qualité nutritionnelle, le jus salé A présente des caractéristiques plus intéressantes par rapport à celle du jus non salé B ; surtout concernant la concentration en sodium et en taux de $\beta$-carotènes (Tableau 2). En effet, le taux en sodium élevé pour le jus salé (Jus A) est dû à l'ajout de sel (1\%). Ceci suggère que l'ajout de sel a une légère influence sur la teneur en $\beta$-carotènes. Toutefois, les études de Bernier et La voie (2001) ont montré que la teneur en $\beta$ carotènes est liée à la variété de tomate et du stade de maturité du fruit. Mais Capanoglu et al. (2008) ont montré que seule l'étape de la concentration de la purée de tomate fait diminuer la teneur en $\beta$-carotènes. Par ailleurs, la concentration du jus salé est proche du jus de tomate assaisonné vendu sur le marché européen qui est de $280 \mathrm{mg} / 100 \mathrm{ml}$ d'après l'Union Nationale Interprofessionnelle des Producteurs de Jus de Fruits en France (Glouchkoff, 2010). L'autre intérêt du jus de tomate est qu'elle est plus riche en vitamine $\mathrm{C}$ et en potassium pour chacun des deux types de jus salé et non salé parce que l'ajout de sel n'a pas eu une influence significative sur ces deux éléments nutritifs. Dans cette étude, la teneur en potassium obtenue pour les deux types de jus est similaire à celle obtenue avec les échantillons du jus de tomate assaisonné et vendu sur le marché (Glouchkoff, 2010). Mais la teneur en vitamine $\mathrm{C}$ du jus de tomate (14 $\mathrm{mg} / 100 \mathrm{ml}$ ) obtenue avec ce même auteur n'est pas similaire aux valeurs de teneur en vitamine $\mathrm{C}(10,46 \mathrm{mg} / 100 \mathrm{ml})$ obtenues dans cette étude. Cet écart de teneur en vitamine $\mathrm{C}$ peut dépendre de la variété de tomate utilisée. Cette variation de la teneur en vitamine $C$ peut être aussi due d'une part aux différentes opérations unitaires de transformation qui ont été réalisées dès la réception des fruits. D'autre part Chanforan (2010) a montré que la teneur en vitamine $\mathrm{C}$ du concentré ou du jus de tomate est surtout influencée par les opérations thermiques. Par ailleurs, les travaux de Agassounon et al. (2012), effectués, sur la tomate, ont révélé que la vitamine $\mathrm{C}$ est une molécule oxydable très vulnérable au traitement à la chaleur (blanchiment).

\section{Conclusion}

Cette nouvelle méthode permet de mieux valoriser la tomate et par conséquent, contribue à la réduction des pertes postrécolte. Le transfert de cette nouvelle méthode de transformation de la tomate au niveau du paysan permettra non seulement de contribuer à la réduction de l'utilisation du bois de feu mais aussi de susciter une autre activité génératrice de revenu qu'est la production et la commercialisation du jus de tomate.

\section{CONFLIT D'INTERET}

Les auteurs de ce manuscrit déclarent que l'article n'est sujet d'aucun problème ou d'intérêt concurrentiel.

\section{CONTRIBUTION DES AUTEURS}

Les auteurs de l'article ont participé chacun en ce qui le concerne à la mise au point du manuscrit. En effet, le travail de paillasse, le protocole donnant le sujet, les objectifs du projet d'article, la rédaction et l'analyse statistique des données a été effectué par PAFH, l'investigateur principal, assisté de $\mathrm{VD}$ et ADA. Le protocole a été mis en exécution par ADA et VD. Enfin la lecture du manuscrit a été faite par LA-F et GAM.

\section{REFERENCES}

Agassounon DTM, Gomez S, Tchobo FP, Soumanou MM, Toukourou F. 2012. Essai de conservation de la tomate par la technique de la déshydratation imprégnation par immersion (DII). Int. J. 
Biol. Chem. Sci., 6(2): 657-669. DOI : http://dx.doi.org/10.4314/ijbcs.v6i2.10

AOAC. 2012. Official Methodsof Analysis $14^{\text {ème }}$ edn, Sidney W (ed). Association of Official Analytical Chemists : Virginie, USA ;22209,

Bernier V, Lavoie D. 2001. Le lycopène: un antioxydant très puissant. Deuxième partie. Le Clinicien, 16(12) : 49-56.

Biehler E, Mayer F, Hoffmann L, Krause E, Bohn T. 2009. Comparison of 3 Spectrophotometric Methods for Carotenoid Determination in Frequently Consumed Fruits and Vegetables. Journal of Food Science, 75(1): 55-61. DOI: $10.1111 / \mathrm{j} .1750-3841.2009 .01417 . x$

Boumendjel MM, Houhamdi MF, Samar H, Sabeg A, Boutebba M. 2012. Effet des traitements thermiques d'appertisation surla qualité biochimique, nutritionnelle ettechnologique du simple, double et triple concentre de tomate. Sciences et Technologies, 36: 51-59.

Boumendjel MM, Boutebba M. 2003. Effet des barèmes de sterilisation sur la qualite biochimique et nutritionnelle des tomates en conserve. Sciences et Technologies, 20: $51-55$.

Capanoglu E, Beekwilder J, Boyacioglu D, Hall R, De Vos R. 2008. Changes in antioxidant and metabolite profiles during production of tomato paste. Journal of Agricultural and Food Chemistry, 56: 964-973.

Chanforan C. 2010. Stabilité des micro constituants de la tomate (composés phénoliques, caroténoïdes, vitamines $\mathrm{C}$ et E) au cours des procédés de transformation : études en systèmes modèles, mise au point d'un modèletoechio-cinétique et validation pour l'étape unitaire de préparation de sauce tomate. Thèse de Doctorat. Université d'Avignon et des pays de Vaucluse, p. 399.

Dossou J, Soulé I, Montcho M. 2006. Analyse économique de la production de purée de tomate à petite échelle au Bénin. Tropicultura, 24(4) : 239-246.
Dossou J, Soulé I, Montcho M. 2007. Evaluation des caractéristiques physicochimiques et sensorielles de la purée de tomate locale produite à petite échelle au Bénin. Tropicultura, 25(2) :119-125.

Glouchkoff A.2010. Composition et profil nutritionnel des aliments. http://www.idietetique.pro. Consulté en juillet 2012.

Kiki D, Fagbohoun O. 1999. Guide pratique: techniques artisanales de fabrication de purée de tomate. Imprimerie Cartographique du CENAP, INRAB/MDR, (Bénin), p. 62.

Kpagbin C. 2011. La conservation de la tomate par séchage et son application sur les deux variétés les plus cultivées au Benin. Mémoire de fin de formation pour l'obtention de diplôme de licence professionnelle. UATM/GASA FORMATION, Cotonou (Bénin), p.60.

Montcho MD. 2002. La transformation de la tomate locale en purée : une approche de solution pour réduire les pertes de production en période de saturation du marché. InRural. Universe Network SPIPM Interactive article $\mathrm{n}^{\circ} 1064$.

Montcho DM, Ahouansou R. 2004. Rapport de test d'évaluation et adaptation d'une épépineuse de tomate destinées à la fabrication de la purée. Rapport de recherche PTAA/INRAB, (Bénin), p.14.

Gueguen L, Rombauts P. 1961. Dosage du Sodium de Potassium, de Calcium et du Magnésium par spectrophotométrie de flamme dans les aliments, le lait et les excréta. Ann. Biol. Anim. Bioch. Biophys, 1(1) :80-97. DOI : http://dx.doi.org/ 10.1051/rnd/19611080

Sawadogo I, Koala M, Dabire C, Ouattara LP, Bazie VBEJT, Hema A, Gnoula C, Pale E, Nebie RHC. 2015. Etude de l'influence des modes de transformation sur les teneurs en lycopène de quatre variétés de tomates de la région du nord du Burkina Faso. Int. J. Biol. Chem. Sci., 9(1): $362-370 . \quad$ DOI : http://dx.doi.org/10.4314/ijbcs.v9i1.31 